Kong. Res. J. 4(2): 73-79, 2017

ISSN 2349-2694

Kongunadu Arts and Science College, Coimbatore.

\title{
CULTIVATION AND ECONOMICAL PERSPECTIVES OF GRACILLARIA: MARINE SEAWEED
}

\author{
Sumayya, S.S ${ }^{1}$., S.G. Sreelekshmi ${ }^{2}$ and K. Murugan* \\ 1Plant Biochemistry and Molecular Biology Laboratory, Department of Botany, \\ University College, Thiruvananthapuram. \\ ${ }^{2}$ SCMS Institute of Bioscience and Biotechnology Research and Development, Kochi. \\ *E.mail: harimurukan@gmail.com
}

\begin{abstract}
For decades, seaweed has been of biological, industrial, and pharmaceutical importance. Because of their nutraceutical potential, seaweed has been used as a food throughout Asia. Traditional Chinese medicine used aqueous hot extracts of certain seaweeds in the treatment of cancer. Further, the Japanese and Chinese cultures have used seaweeds to treat goiter and other glandular problems since $300 \mathrm{BC}$. The Romans used seaweeds in the treatment of wounds, burns, and rashes. The Celts noted that ordinary seaweed contracted as it dried and then expanded with moisture. In Scotland during the 18th century, physicians used dried seaweed stem to successfully drain abdominal wall abscesses. They also inserted seaweed into the cervix in an attempt to treat dysmenorrhea. Many reports outline the use of seaweed to induce abortion. Seaweed was employed intravaginally for ripening of the cervix and was used rectally for strictures. In this juncture, culture and therapeutic potential of Gracilaria was reviewed. Gracilaria is a genus of red algae notable for its economic importance as an agarophyte, as well as its use as a food for humans and various species of shellfish. Various species within the genus are cultivated among Asia, South America, Africa and Oceania. Gracilaria is used as a food in Japanese, Hawaiian, and Filipino cuisine. In Japanese cuisine, it is called ogonori or ogo. In thePhilippines, it is called gulaman and used to make gelatin. In Jamaica, it is known as Irish moss. The moisture content is $12 \%$ and protein is $8 \%$. The species are used by local people as salad, preparation of various curries and industrially many by products are synthesized from this sea weed. Medicinally as microbicidal, antiinflammatory, antimetastatic and immuno modulatory potential.
\end{abstract}

Keywords: Gracilaria, seaweeds, culture, medicinal, nutraceutical.

\section{INTRODUCTION}

Gracilaria species are common warm water seaweeds. There are more than one hundred species in the world, some of which have very important economic value. Gracilaria is used as food and in the preparation of food products.

In China, Gracilaria species were used as food and as binding material in the preparation of lime for painting walls. In course of time it was popularized in several Asiatic countries, until its content of agar were discovered by the Japanese and the Western countries. A diversity of cultivation methods has been developed in different places, which is also consistent with the great variety of species of gracilarioids and their great range of temperature and latitudinal tolerance (Hansik and Ryther; 1984).

In the late fifties to early sixties, commercial cultivation was carried out in the Guangdong Province of China by inserting branches of the seaweed into splits of bamboo sticks about $15 \mathrm{~cm}$. long, which were then planted in littoral farms. Later, cultivation of Gracilaria was carried out by scattering juvenile thallus fragments $(5-6 \mathrm{~cm}$. long) in littoral farms sheltered from wave action. Thalli in the fish ponds were covered with old fishing nets to avoid drifting. The method was initiated in Taiwan and by the late seventies, the Gracilaria ponds occupied over 200 hectares in area with production reaching over 6800 tonnes and worth almost US\$ 4.7 million. Experiments on growing Gracilaria in ponds or lagoons were later done in Brazil, Italy, Sri Lanka, Malaysia, Philippines and Japan. The method has proved to be successful in Taiwan province of China, China, Vietnam, Thailand and Indonesia. Two cultivation methods of Gracilaria were developed in the 1980s. In St. Lucia, Lesser Antilles, Gracilaria was successfully cultivated on lines suspended near the surface by floating bamboo. These were later replaced by plastic bottles. A system of suspended cultivation with ropes and buoys was later developed in Namibia, while experiments with suspended ropes have been made also in Cuba, South Africa, India, the Philippines and New Zealand. At present, the most successful cultivation system with ropes and significant economic returns is being 
developed in China with Gracilariopsis lemanaeformis (Santelices and Doty; 1989).

During the 1980s, a different cultivation method was developed in Chile. Because the thallus can survive burial in sandy mud for month, thallus fragments can be pushed in the mud with a fork or held down with sand-filled polyethylene tubing until the plants are established and growing. Harvesting is by hand, taking care not to remove the underground thalli. This method is the basis of the commercial production of Gracilaria chilensis and is confined to sheltered intertidal and shallow subtidal muddy sandy areas. Experimental tank cultivation of Gracilaria also has proved to be technically feasible, first in Florida for energy and later in Sweden, to improve agar quality and quantity (Ohno and Critchely; 1993).

\section{MAJOR PRODUCTS}

The FAO statistics for several species of Gracilaria are combined within the map shown below. Data are separated for what FAO has called Gracilaria spp. and warty Gracilaria. The last taxon is supposed to correspond to $G$. verrucosa, (now $G$. gracilis) being produced in China, Taiwan Province of China and other places of Asia and amounting to some 1.5 million tonnes. Gracilaria spp. includes all other species, being produced in Africa (Namibia and South Africa), America (Argentina, Brazil, Chile and Peru), Asia (Indonesia, Korea Philippines and Viet Nam). Not all of them, have productions registered in the national statistics. By 2011, the total production of Gracilaria spp. amounted to 700000 tonnes (Bird and Porse; 1984).

\subsection{Biology of Gracilaria}

The typical habitats of these species are sheltered environments such as bays, estuaries, or river mouths. They grow on intertidal or subtidal rocky, sandy or sandy-muddy substrates, or on rocky outcrops associated with sandy beaches. They can be intertidal or sub tidal, down to $20 \mathrm{~m}$ depth, attached to small stones, partially covered by sand or anchored in sand. Often in areas with good water circulation. The fertilization of the egg in the carpogonium of the female gametophyte by the spermatium results in the production of the zygote, which in turn develops in the macroscopic carposporophyte within the fertile structure called cystocarp in the female gametophyte.

The carpospores $(2 \mathrm{n})$ produced by the carposporothyte develop into the tetrasprophyte. Meiosis takes place in the tetrasporangia, resulting in the production of tetraspores (n) which in turn develop into the gametophytes. The gametophyte and the sporophyte are the large phases in the life cycle of this seaweed. These seaweeds are characterized by their high vegetative regenerative capacity, a characteristic used by farmers to propagate the crop.

\section{CULTIVATION}

Given the diversity of cultivation methods used in the production of Gracilaria spp., the four most important are described below (Shang; 1976).

\subsection{Pond Farming}

Supplies of seed stocks are sourced from the wild, from crops obtained in other ponds or from nursery-reared cuttings. Ponds are generally located in areas not exposed to strong prevailing winds, near sources of freshwater and seawater. Usually pond depth is kept at $30-40 \mathrm{~cm}$ and water is exchanged every 2-3 days. Salinity is adjusted by freshwater additions while expected seasonal temperature regimes can be modified by pond depth (deeper ponds in warmer areas or seasons). Mineral, especially nitrate and phosphates can be added for seaweed growth. Stocking densities are 5-6 tonnes /ha. After 30-35 days of growth in summer and 4045 days in winter, harvesting is undertaken removing one third to half of the total biomass. Annual production figures of about 34 tonnes / ha have been reported for this production system.

\subsection{Rope Farming}

Two methods of out-planting using ropes are utilized to grow Gracilaria. One starts from vegetative materials that are tied or inserted within a rope. The supplies of seed stocks also may be sourced from the wild, from crops obtained in previous cultivation or from nursery-reared cuttings. The second method involves reproductive materials which are used as a source of spores which are left to settle on the surface of the ropes. The ropes or lines used can be monofilament, nylon or other suitable line. Durability of the line in seawater should be tested before large quantities of line are purchased. The rope can be opened to insert the Gracilaria fragments or they could be tied at regular intervals to the main rope using pieces of raffia or tape. Once the Gracilaria is attached, ropes are then suspended, stretched between stakes buried in the sediment or supported at different levels by buoys or rafts. Light intensity and transparency of the water column are important factors that may limit survival and growth of the species under cultivation. Field conditions are site and species specific and should be tested previously to large-scale investment on farming. Spores may be set onto lines, allowing them to settle on the lines after laboratory induction of spore 
release or lying ropes as substratum for spores in natural populations of fertile Gracilaria. Rope farming results in different levels of production, depending on climatic conditions and the species being cultivated. In Ceylon, up to $3.5 \mathrm{~kg}$ per m-1 could be obtained per crop while in China, productions up to 2 tonnes per ha-1 (dry) are reported.

\subsection{Bottom stocking}

The simplest method is to transfer rocks bearing the thalli to places where an increase in density is wanted. If the species is already growing on a site, this labor-intensive method is often successful. A more complex procedure is to remove thalli or major branches and put them in places where they are wanted. Two further common approaches are to tie the thalli to rocks, or to secure them to rocks by means of rubber bands. Another method used on non-consolidated bottoms is to push the proximal ends of whole thalli or major branches into the planting site bottom. This is feasible and is used in Chile with some promise. It is especially useful for intertidal mud flats where farmers can easily push bundles of thalli into the sand or mud during low tides. However, materials planted in substrate may tear loose during harvesting or during periods of much water movement. This problem has been common in the shallow intertidal flats of southern Chile. In addition planting and harvesting are high labor-consuming activities which can be economic only in areas with low labor costs (Zemekewhite, 2004).

A more elaborate system has been designed for subtidal beds on sandy bottom. Gracilaria is anchored using soft polyethylene tubes $1 \mathrm{~m}$ long, 0.1 $\mathrm{mm}$ thick and about $4 \mathrm{~cm}$ in diameter, which are completely filled with dry sand (ca. $2.5 \mathrm{~kg}$ ) and knotted at both ends. Keeping them wet and cool, apical and middle portions of thalli about $40 \mathrm{~cm}$ long, collected from natural beds, are secured to the sandfilled polyethylene tubes with rubber bands. Five to six strands of the Gracilaria (about $90 \mathrm{~g}$ ) are tied along one side of each anchoring unit. These units are then placed on a boat, transported to the planting area, dropped from the boat and arranged underwater by divers. Normally they are set in parallel rows, about $1 \mathrm{~m}$ apart and positioned perpendicular to the coastline. When setting up the rows, precautions are taken to rest the centers of the bundles directly on the substratum, where they are held in place by the weight of the sand in the tubes. Experimental evaluation of the polyethylene tube method has suggested potential yields of about 21 tonnes (dry) per ha for a 6 months growing period (Fredericq, 1989).

\subsection{Tank Farming}

Growing Gracilaria in tanks permits control over the whole production process. Also the method has the promise of achieving more sophisticated ends, e.g., the processing of polluted water to obtain some specific material (e.g. agarose) in addition to clean water. This method is particularly attractive in high-labor-cost areas, where capital returns are the principal benefits. Also, it provides the greatest productivity per unit area, much greater than any other type of farming (some 127 tonnes per ha per year). Efficiency of the system is dependent upon the input of various types of energy (compressed air for bubbling, carbon dioxide and pumping of water). Influent water should be filtered or held in sedimentation tank to reduce the problem of siltation in the tank. The tank cross-section may be $\mathrm{v}$ - or $\mathrm{u}$-shaped and constructed from any number of convenient materials. Aeration systems are provided using air compressor and perforated PVC pipes.

For several reasons not completely understood, tank yields have been found to be directly proportional to seawater exchange rates of between 1 and 30 culture volumes/day. Carbon dioxide addition and $\mathrm{pH}$ adjustments can substitute for up to ten water exchanges per day. Under low water flow, Gracilaria growth may be limited not only by mineral nutrient availability but also by $\mathrm{CO} 2$ limitations. Accepting this, high production of Gracilaria in a land-based pond or raceway culture system would be carbon limited; the solution would be to add large volumes of water or large amounts of $\mathrm{CO} 2$ gas to the culture. In addition, there must be sufficient aeration to maintain the seaweed in suspension and to rotate it. Periodic aeration (15 min per hour, for a total of $7 \mathrm{~h}$ a day) proved to be as efficient as continuous aeration. Increases in duration of aeration up to $24 \mathrm{~h}$ per day were accompanied by increases in productivity but at such cost that little economic gain occurred after about 12 $\mathrm{h} ; 11 \mathrm{~h}$ daily appeared to be the most economical.

Contrary to common expectations, continuous mineral nutrient additions to the tanks resulted in a reduction in the growth of Gracilaria due to enhanced growth of epiphytes (Kain;2003). However, two biological findings suggested an optimum nutrient management program for Gracilaria. First, it was found that epiphytes such an Enteromorpha take up nitrogen mainly in response to light, while Gracilaria takes up nitrogen at night as well as by day. Secondly, seaweeds have the capacity to store mineral nutrients when external supplies are available and then draw upon these reserves when external concentrations are low. Therefore, if 
fertilizing is done only at night, and at 3 to 6 day intervals, such green algae as Enteromorpha may not become a pest. Nutrient supply to cultures has been successfully provided by pulse feeding of various concentrations at suitable frequencies.

The Gracilaria crop should be harvested periodically to maintain a high level of productivity. If the standing crop became too thick, self-shading may result. The harvesting methods vary depending on the size of the tanks and harvesting frequency. It may be adequate to use hand nets. Larger operations may require continual removal by mechanical scoops. Tanks could be drained into a net but efficiency might be compromised by the time needed to refill the tank.

Although the sustained yield of Gracilaria cultivated in tanks is among the highest for any seaweed tested, this production system for biomass is economical for a few types of production only (e.g. Gracilaria as fresh food). The method of cultivation employed is very energy intensive because it requires large amounts of flowing seawater and aeration. In addition, in many developed nations the requirement of large acreages of coastal land for land-based tank or raceway systems seems to be economically prohibitive. The FAO reported statistics for this group of seaweeds separate the warty Gracilaria from the other species of Gracilaria (Gracilaria spp.). The warty Gracilaria is supposed to correspond to Gracilaria verrucosa. This is an invalid name and the species now is recognized as Gracilaria gracilis. Since G. verrucosa was a common name in the past, many of the classical reports might be referring to different species (Patra and Muthunaman et al., 2013).

Landing of the two types of Gracilaria distinguished have steadily increased over the last decade. By 2011, about 2257919 tonnes of Gracilaria were reported. The totality of what is recognized as warty Gracilaria (1 518455 tonnes) is produced by cultivation mainly in China (1 513590 tonnes) and Taiwan province of China (some 4865 tonnes). In the case of Gracilaria spp., 94.2 percent of the production (some 697240 tonnes) is by cultivation while the remaining (42 224 tonnes) are gathered from wild stocks. The most productive countries in America are Chile, Perú and Argentina. In Asia, there is productive cultivation of Gracilaria is Indonesia, Vietnam, the Philippines and Korea while in Africa only Namibia reports Gracilaria production.

The principal product of Gracilaria is agar. Industrial applications are dominated by three quality grades, a) sugar reactive agar, b) standard agar and c) food-grade agar. In the sugar reactive agar, the gels are stronger as a function of sugar concentration. It is obtained largely from Gracilariopsis lemaneiformis, at present the most important species under cultivation in China. Standard agar is recognized because the gel has the temperature, consistency and structure for microbiological purposes. It is produced largely by other seaweed groups such as Gelidium, Pterocladia or Pterocladiella. The food grade agar designates any kind of agar not meeting the requirements for sugarreactive or bacteriological agar. It is extracted from a wide variety of Gracilaria species (Kumar et al; 2013).

Market demands for species of Gracilaria to produce agar have increased markedly in a decade (1999-2009). Table 2 summarizes several important market parameters. Global agar production increased from 7500 to 9600 tonnes, with sale prices of USD $17 / \mathrm{kg}$ increasing, on average, to USD $18 / \mathrm{kg}$. The world agar sale value increased, therefore, from USD 128 million in 1999 to USD173 million in 2009. In 1999, about 63 percent of the total agar production was produced by Gracilaria. In 2009, the relative importance of Gracilaria had increased to 80 percent of the total agar production. These data indicate that although the industrial agar growth has been modest, it has been enough to generate the cash flows necessary to support the overheads needed for regulatory reform and capital investment needed to improve plants and equipment. Selling price increases have generally been adequate to offset seaweed, energy and chemical costs. In addition, the cultivation of Gracilaria has provided enough raw materials to support expansion. In fact, Gracilaria has grown in importance for extracting agar between 1999 and 2009 while Gelidium is declining in importance (Luhan; 1996).

Unknown quantities of Gracilaria are used in several places (e.g. Japan, China, Hawaii, St. Lucia) in the fresh vegetable market for human consumption. There, Gracilaria prices can be high (e.g. 5-7 USD $/ \mathrm{kg}$ ) although the volumes consumed are relatively low (e.g. a few tonnes per year).

The use of Gracilaria as marine invertebrate feed has developed in Asia, especially in the fish pond system of Taiwan Province of China, leading to development of polycultures of Gracilaria and abalone which uses Gracilaria as the sole source of food of these gastropods. In the past the emphasis was to produce Gracilaria biomass for agar production. Now it is more profitable for the pond operators to supply their Gracilaria as fresh food to abalone farmers (Bixler and Porse; 2011). 


\section{OTHER USES}

Intensive research over the last decade is showing several potential economic uses for different species of Gracilaria and Gracilariopsis. For sake of clarity, the findings have been grouped in four main uses. However, it is clear that with adequate technology several of these uses could be accomplished reutilizing the same material.

\subsection{Paper making}

During the agar extraction process from Gracilaria or Gracilariopsis, considerable amounts of solid residues are produced as extraction wastes. The potential for using agar extraction residues as raw materials for pulping and paper making has been explored in China (Pei et al., 2013) finding that the extraction wastes indeed could be utilized for papermaking (e.g. as a fiber source, as a functional filter, etc.). The higher contents of algal materials in the hand sheets samples resulted in lower permeability and stronger antimicrobial effects than the common paper. Algal material, when used as a partial substitute for wood pulp, resulted in improved paper density, waterproofness, grease proofness, and antimicrobial effects, indicating its potential use in the food packaging industry.

\subsection{Biofuel}

The large carbohydrate contents of same species of Gracilaria (about 45 percent of dry weight; Amanullah et al., 2013) and which is normally used as agar or food, could have an alternative use for ethanol production. Amanullah et al. (2013) recently demonstrated such possibility using field cultivated Gracilaria edulis and fermenting its polysaccharides to ethanol, using Saccharomyces cerevisiae in the fermentation process. In a related study Kumar et al. (2013) developed an efficient strategy for agar extraction using the resultant pulp for bioethanol production. Thus, they suggested an integrated biorefinery process as the kind of activity most likely to obtain maximum economic returns from Gracilaria. Furthermore, they noticed that after ethanol production the leftover residues still contained good amounts of organic matter and useful minerals, and eventually could be used as biofertilizer.

\subsection{Multi-products source}

A diversity of studies on chemical composition of species of Gracilaria and on the effects of some of its many bioactive compounds are revealing a large variety of compounds and effects (Francavilla et al., 2013; Patra and Muthunaman, 2013; Tabarsa et al., 2012) to the point of suggesting some of the species of this genus could be characterized as a multiproduct source for biotechnological, nutraceutical and pharmaceutical applications even though more investigations are required for separating, purifying and characterizing many of these compounds.

\subsection{Bioremediation capacity}

The capacity of seaweeds to remove inorganic nutrients from the water media has been recognized for many decades. In present day integrated multitrophic aquaculture, the species of seaweeds are viewed (Chopin et al., 2001) as renewable biological nutrients scrubbers that take up nutrients. Various species of Gracilaria have been evaluated in their removal capacity of nutrients produced from invertebrate or fish farms. For example, Troell et al., (1997) concluded that a suspended culture of 1 ha, at a stocking density of 1 $\mathrm{kg}$ wet weight $\mathrm{m} 2$, removes 5 percent of the dissolved inorganic phosphorus released from a 227tonnes mixed salmon farm. Thus different species of Gracilaria are being considered as good candidates or the establishment and exploitation of multithrophic cultivation systems.

\section{STATUS AND TRENDS}

Several fronts of active research are focusing on Gracilaria spp. For example, there are renewed efforts to characterize species and strains of productive taxa, using molecular analysis. The final goal is to maintain culture collections of the most productive species, strains and cultivars perfectly identified as to trace their distribution and genetic changes over time and space.

Closely related to the above goal is the present concern to learn on the genetic diversity of cultured stocks. Several studies are showing that cultured populations exhibit reduced genetic diversity which seemingly results in production decline and increased frequency of outbreaks of pests and pathogens. Studies are oriented to analyze genetic diversity of cultured stocks as well as to obtain genetic improvement.

The effect of various environmental factors on growth and propagation of different species as well as on the quantitative and qualitative characteristics of its agar continues to be a popular subject. The effects of irradiance, salinity, U.V., temperature and different types of nutrients are being studied with different life cycle stages of species such as Gracilaria edulis, G. tenuistipitata, G. changii and G. chilensis. This combination of studies are gradually broadening the basis for successful 
cultivation and farming of economically interesting species in various geographic areas.

Several types of studies are evaluating developments that could increase the consumption of Gracilaria and its agar. For example, development of new products are anticipated to be the factor that could substantially modify the market, perhaps allowing for better product differentiation. Better raw material management would increase higher yields in agar production. Facing the climatic changes ahead, there is a need for more industry/government sponsored research into strain improvement that might be more tolerant of environmental changes.

It is also recognized that in the future, food applications will continue to increase driven by the growth of processed foods in developing countries. Therefore, the farming of species highly productive for food-grade agar will have to be increased or domestication and cultivation of new species will be needed. The development of seaweed farming may have negative and positive effects. The development of seaweed farms inevitably involves alteration of natural habitat. This may include alteration of the substratum or removal of other seaweed species or invertebrates to give way to the farm. In many places there is a complete absence of studies addressing this problem and its consequences. Often the farming communities lack the capacity to evaluate the changes or to figure out the long-term ecological consequences of these changes. In other cases, the economic benefits of Gracilaria farming have quickly overcome any possible concern for environmental impact. In countries and regions with enough economic development (e.g. USA, New Zealand, and Australia) visual obstructions that will lower the value of coastal properties or conflict of interest with recreational uses of the sea have limited the development of farming areas.

Seaweed in a farm can sometimes utilize the nutrients from an environment that already might not be very rich in nutrients, causing nutrient depletion. In addition, the invertebrates and fishes (herbivores) that might increase in density during intense algal growth, probably suffers significant reductions after seaweed harvesting.

A final aspect to be considered is the significant habitat modification that might be produced by the introduction of farming implements, such as plastic tubes (in the bottom farming system), monolines, plastic straw used to tie the seaweed, bamboo sticks and similar. Whenever farming stops for whatever reason, farming refuse (e.g. old monolines and so on) often are abandoned in the area without attempts to clean the contaminants or to restore the original habitat.

On the positive effects of farming, seaweeds may be used as nutrient sink, especially relevant in association with invertebrate farming or multitrophic types of farming. Also they can increase primary productivity and to expand the habitat area and food supply for herbivorous fish and invertebrates.

\section{REFERENCES}

Amanullah, T.R., D. Krishnan, D.S. Balaji, K. Ganesan, B.G. Anand, I.S. Barthelomai, R. Ravichandran and S.K. Chinnasamy.(2013). Effective production of ethanol-from-cellulose (EFC) from cheap sources sawdust and seaweed Gracilaria edulis. Advances in Applied Science Research, 4(5):213-222.

Bird C.J., and J. Mc Lachlan, (1984). Taxonomy of Gracilaria: evaluation of some aspects of reproductive structure. Hydrobiologia, 117: 4146.

Bixler H.J., and H. Porse,(2011). A decade of change in the seaweed hydrocolloids industry. Journal of Applied Phycology, 23: 321-335.

Chopin T., Buschmann A.H., Halling C., Troell, M., Kautsky, N., Neori, A., Kraemer, G., ZertucheGonzalez, J., Yarish, C., Neefus, C., (2001). Integrating seaweeds into aquaculture systems: a key towards sustainability. Journal Phycology, 37:975- 986.

Francavilla M., Franchi M., Monteleone $M$ and Caroppo C. (2013). The Red Seaweed Gracilaria gracilis as a Multi Products Source. Mar. Drugs, 11: 3754-3776.

Fredericq S., and Hommersand M.H., (1989). Proposal of the Gracilariales ord. nov.(Rhodophyta) based on an analysis of the reproductive development of Gracilaria verrucosa. Journal of Phycology, 25: 213-227.

Hanisak M.D. and Ryther J.H., (1984). Cultivation biology of Gracilaria tikvahiae in the USA. Hydrobiologia, 117: 295-298.

Kain J.M., (2003). Seaweed cultivation in the West is retarded. In, T.A. Norton (Ed.) Out of the past. British Phycological Society. Belfast.pp:163-180.

Kumar S., Guptab R., Kumar G., Sahooa, D., Kuhadb R.C., (2013). Bioethanol production from Gracilaria verrucosa, a red alga, in a biorefinery approach. Bioresource Technology. 135: 150156.

Luhan M. R. J., (1996). Biomass and reproductive states of Gracilaria heteroclada Zhang et Xia 
collected from Jaro, Central Philippines. Bot. Mar. 39: 207-211.

Ohno M., and Critchley A.T., (1993). Seaweed cultivation and marine ranching. Japan International Cooperation Agency, pp: 151.

Patra S., and Muthuraman M.S., (2013). Gracilaria edulis extract induces apoptosis and inhibits tumor in Ehrlich Ascites tumor cells in vivo. BMC Complementary and Alternative Medicine. 13:331-343.

Pei Jicheng., Lin Apeng., Zhang Fangdong., Zhu Daling., Li Jian., Wang Guangce., (2013). Using agar extraction waste of Gracilaria lemaneiformis in the papermaking industry. Journal of Applied Phycology, 25 (4): 1135-1141.

Santelices B., and Doty M.S., (1989). A review of Gracilaria farming. Aquaculture 78: 98-133.
Shang Y.C., (1976). Economic aspects of Gracilaria culture in Taiwan. Aquaculture 8: 1-7.

Tabarsa M., Rezaei M., Ramezanpour Z and Waaland R.J.,(2012). Chemical compositions of the marine algae Gracilaria salicornia (Rhodophyta) and Ulva lactuca (Chlorophyta) as a potential food source. Journal of Science Food and Agriculture. 92: 2500-2506.

Troell M., Halling C., Nilsson A., Buschmann A.H., Kautsky N., Kautsky L., (1997). Integrated marine cultivation of Gracilaria chilensis (Gracilariales, Rhodophyta) and salmon cages for reduced environmental impact and increased economic output. Aquaculture. 156, 45- 61.

ZemkeWhite W.L., (2004). Assessment of the current knowledge of the environmental impact of seaweed farming in the tropics. Pp: 465-476. 